\title{
Covert orienting: A compound-cue account of the proportion cued effect
}

\author{
Evan F. Risko, Chris Blais, Jennifer A. Stolz, and Derek Besner \\ University of Waterloo, Waterloo, Ontario, Canada
}

\begin{abstract}
An increase in the proportion of spatially cued trials in the context of the covert orienting paradigm increases the magnitude of the cuing effect. This proportion cued effect is widely interpreted to reflect a form of control. Specifically, it is argued that participants strategically allocate attention as a function of the utility of the spatial cue. Here, an alternative explanation of the proportion cued effect is proposed that does not require control. According to this account, the cue-target event forms a compound cue and the proportion cued manipulation produces a relative disparity in the frequency with which particular compound cues occur. Specifically, when the proportion of spatially cued trials is increased, the frequency of spatially cued cue-target events increases and the frequency of spatially miscued cue-target events decreases, thus increasing the magnitude of the cuing effect. The results of two experiments support this account.
\end{abstract}

In the covert orienting paradigm, a spatial cue (e.g., an arrow or a peripheral onset) is used to direct the participant's visual attention to a particular location before a target is presented (Posner, Nissen, \& Ogden 1978). When attention is directed to the upcoming target location (a spatially cued trial), responses are faster than when attention is directed to a nontarget location (a spatially miscued trial). Changes in the magnitude of the cuing effect (the difference in response times on spatially cued and miscued trials) resulting from the manipulation of various factors form the empirical foundation on which extant theories of visual attention are largely based (e.g., Posner \& Petersen, 1990). In the present investigation, we take one such empirical phenomenon - the effect of proportion cued on the magnitude of the cuing effect - and propose an alternative to the long-held interpretation of this effect. We also provide experimental evidence consistent with this proposal.

\section{The Proportion Cued Effect}

As the proportion of spatially cued trials in a covert orienting experiment increases, the magnitude of the cuing effect also increases (e.g., Eriksen \& Yeh, 1985; Johnson $\&$ Yantis, 1995; Madden, 1992). The most widely accepted explanation of this proportion cued effect is that participants are strategically controlling the distribution of visual attention in response to the utility of the spatial cue. According to this account, as the utility of the spatial cue increases (i.e., as the proportion of spatially cued trials increases), participants allocate increasing amounts of attention to the spatially cued location. As a result, responses become faster on spatially cued trials and slower on spatially miscued trials, thereby producing a larger cuing effect.
This control account of the proportion cued effect reflects the received view in the attention literature. Indeed, researchers have gone so far as to use the presence/absence of a proportion cued effect to make inferences about the ability to shift attention in a controlled manner (Danckert, Maruff, Crowe, \& Currie, 1998; Enns \& Brodeur, 1989; Maruff, Danckert, Pantelis, \& Currie, 1998). For example, Enns and Brodeur took the lack of a proportion cued effect in children 6-8 years old as evidence for a strategic deficit in young children's ability to allocate attention.

The control account of the proportion cued effect is intuitively appealing; however, we demonstrate here that it is not the only plausible explanation. In what follows, we develop an alternative account of the proportion cued effect that does not attribute it to a control mechanism. We then demonstrate the viability of this account in two experiments.

\section{The Compound-Cue Frequency Account of the Proportion Cued Effect}

What we call the compound-cue frequency account of the proportion cued effect is based on a well-established empirical observation coupled with a novel application of a theoretical idea developed in the context of research on memory retrieval. The first part is that it is well-known that frequency of occurrence is negatively correlated with response time (e.g., Bertelson \& Tisseyre, 1965; Hyman, 1953). For example, in the context of a discrimination task, when one target occurs more frequently than another, response times to the frequent target are faster than response times to the infrequent target (e.g., LaBerge, Legrand, \& Hobbie, 1969). The second part of the compound cue frequency account is that, in the context of the

E. F. Risko, efrisko@watarts.uwaterloo.ca 
covert orienting paradigm, the cue-target event (i.e., the spatial cue, the target, and the relation between the spatial cue and target) forms a compound cue. According to compound cue theories of memory retrieval, the cue used to access memory consists of a combination of the nominal target and its context (Dosher \& Rosedale, 1997; Ratcliff $\&$ McKoon, 1988). For example, in a semantic priming experiment in which a participant is shown the prime word doctor followed by the target word nurse, the compound cue DOCTOR-NURSE is used to access memory (Ratcliff $\&$ McKoon, 1988). This view can be contrasted with the proposal that only the nominal target serves as the cue for retrieval (e.g., NURSE).

In the context of the covert orienting paradigm, the compound cue would represent some combination of the spatial cue and target. For example, if the participant's task is to discriminate between two targets (e.g., T1 and T2) that are preceded by a spatial cue in one of two locations (e.g., L1 and L2), a compound cue (e.g., T1+L1) rather than only a single cue (e.g., T1) would be used to retrieve a response from memory. The idea that a cue-target relation can be used to support response selection in the covert orienting paradigm has been well-documented (e.g., Kingstone, 1992; Kingstone \& Klein, 1991). For example, Kingstone and Klein demonstrated that violating the expectation that a spatial cue and target will appear in the same location can influence the expectation that a particular target will be presented.

If the cue-target event is represented in memory in some form and can be retrieved via probing memory using a representation of the cue-target event (i.e., using the cue-target event as a compound cue for retrieval), the frequency with which a given cue-target event occurs should influence performance (e.g., Logan, 1988). More frequent cue-target events should be easier to retrieve. In the context of the proportion cued effect, when the proportion of spatially cued trials increases, the frequency of spatially cued cue-target events increases, and, therefore, response times on spatially cued trials should decrease. In a similar vein, when the proportion of spatially cued trials increases, the frequency of spatially miscued cuetarget events decreases and, consequently, response times on spatially miscued trials should increase. The decrease in response times on spatially cued trials and the increase in response times on spatially miscued trials would produce a larger cuing effect. Thus, according to the compound-cue frequency account, the proportion cued effect reflects the well-documented observation that frequent events are responded to more quickly than infrequent events (Bertelson \& Tisseyre, 1965; Hyman, 1953).

Although the effect of frequency of occurrence is well established, the idea that the cue-target event forms a type of compound cue whose frequency affects performance is more controversial. Thus, demonstrating this is critical to the account proposed here. We test this hypothesis in the following experiments.

\section{EXPERIMENT 1}

In Experiment 1, on each trial participants responded whether a single target that appeared either above or below a central fixation was a percent sign or a number sign. Targets were preceded by an abrupt onset spatial cue. Critically, this spatial cue was presented to the left or right of fixation. Thus, the spatial cue provided no information with respect to target location. Cue-target event frequency was manipulated by changing the relative frequency with which the spatial cue appeared in the left or right location. Thus, one cue-target event was more frequent than the other cue-target event. Each target appeared equally often above or below fixation following the left and right spatial cues. Thus, we have a condition in which the frequency of a specific cue-target event is manipulated without also manipulating the utility of the spatial cue.

According to the compound-cue account, responses to frequent cue-target events should be faster than responses to infrequent cue-target events. This neutral-cue frequency effect should occur despite the fact that the spatial cue, overall, provides no information about the task (i.e., it does not predict target identity or location). Because targets appear equally often and are equally likely to follow any cue, if the cue-target event does not act as a compound cue, or the frequency a given cue-target event does not influence retrieval, there should be no difference in response times between frequent and infrequent cue-target events.

\section{Method}

Participants. Forty-eight undergraduate students were paid $\$ 4$ each for their participation.

Design. The experiment consisted of a 2 (frequency: frequent cue-target event vs. infrequent cue-target event) within-subjects design. Cue-target event frequency was manipulated by presenting the spatial cue on one side of the display more often than on the other side of the display. For half of the participants, the spatial cue was presented on the left for $75 \%$ of the trials and on the right for $25 \%$ of the trials. The other half of the participants received the reverse order of presentation.

Apparatus. E-Prime (Psychology Software Tools, 2002) software controlled timing, presentation of stimuli, and logged responses. Stimuli were presented on a 17 -in. monitor.

Stimuli. The stimulus display consisted of a fixation $(+)$ at the center of the screen. A spatial cue appeared $3.6 \mathrm{~cm}$ to the left or right of fixation and consisted of a silver rectangle that subtended $1.0 \mathrm{~cm}$ horizontally and $1.5 \mathrm{~cm}$ vertically. The target was either a percent sign (\%) or a number sign (\#). Both subtended $0.5 \mathrm{~cm}$ horizontally and vertically and appeared $2.5 \mathrm{~cm}$ above or below fixation. Fixation and targets were presented in white on a black background.

Procedure. Participants were instructed that on each trial a target would appear above or below fixation and that they were to respond to the identity of the target by pressing its assigned key. The " $\mathrm{c}$ " and " $\mathrm{m}$ " keys were used as responses. Response-to-target assignments were counterbalanced.

Each trial began with the presentation of the fixation, for a variable amount of time ranging from 500 to $875 \mathrm{msec}$ in $25-\mathrm{msec}$ increments. Variable fixation durations were used to make it more difficult for participants to anticipate spatial cue onset. The spatial cue was then presented for $50 \mathrm{msec}$, and $100 \mathrm{msec}$ after the offset of the spatial cue the target was presented for $150 \mathrm{msec}$. The screen remained blank until a response was made. Participants performed 256 experimental trials preceded by 16 practice trials.

\section{Results}

Response time (RT) analysis was conducted for trials in which the response was correct. These data were subjected to a recursive outlier-trimming procedure that removed 
$2.3 \%$ of the RT data (Van Selst \& Jolicœur, 1994). The condition means for Experiment 1 are presented in the left panel of Figure 1.

A repeated measures ANOVA comparing frequent cue-target events to infrequent cue-target events was conducted for both RTs and percent error. The effect of frequency was significant for RT $[F(1,47)=18.47$, $\left.M S_{\mathrm{e}}=241.89, p<.05\right]$. Responses to frequent cuetarget events $(504 \mathrm{msec})$ were faster than responses to infrequent cue-target events $(518 \mathrm{msec})$. Thirty-five participants demonstrated this effect. There was no effect for errors $(F<1)$.

\section{Discussion}

In Experiment 1, frequent cue-target events were responded to faster than infrequent cue-target events. This neutral-cue frequency effect provides evidence that the cue-target event can be used as a compound cue and that the frequency of that compound cue influences performance. In Experiment 1, despite the fact that the location of the spatial cue is irrelevant to the participant's task, the frequency with which the spatial cue appeared in either the left or the right location influenced performance. This result is difficult to explain if the target acted as the only cue for memory retrieval, but is readily explicable if the cue-target event forms a compound cue. If the cue-target event is used to probe memory, the difference in the frequency with which a specific cue-target event is presented will influence response time. ${ }^{1}$

An alternative account of the results from Experiment 1 is that cue-target event frequency affected the spatial cue's ability to capture attention, in such a way that infrequent spatial cues were more likely to draw attention to their location than frequent cues. Given that the spatial cue never appeared in the target location, if the infrequent spatial cue better captured the participant's attention this would lead to slower responses following infrequent cue-target events than following frequent cue-target events. Experiment 2 was conducted to address this possibility.

\section{EXPERIMENT 2}

In Experiment 2, we used a traditional spatial cuing manipulation in conjunction with a manipulation of cuetarget event frequency. Thus, spatial cue condition (i.e., spatially cued vs. spatially miscued) and cue-target event frequency were factorially manipulated. The compoundcue account and the attention capture accounts of the neutral-cue frequency effect make different predictions about performance on spatially cued trials. According to the compound-cue account, frequent cue-target events should be responded to faster than infrequent cue-target events on spatially cued trials. This is because retrieval is facilitated for frequent cue-target events. The attention capture account makes the opposite predictionspecifically, that infrequent cue-target events should be responded to faster than frequent cue-target events on spatially cued trials. This is because, according to the attention capture account, infrequent cue-target events capture attention better than frequent cue-target events, which would produce a benefit on spatially cued trials. On spatially miscued trials, both the compound-cue account and the attention capture account make the same prediction: Responses to frequent cue-target events should be faster than responses to infrequent cue-target events.

\section{Method}

Participants. Forty undergraduate students were paid $\$ 4$ each for their participation.

Design. The experiment consisted of a 2 (frequency: frequent cue-target event vs. infrequent cue-target event) $\times 2$ (spatial cue condition: spatially cued vs. spatially miscued) within-subjects design. Cue-target event frequency was manipulated by presenting the spatial cue in one location more often than in the other location. For

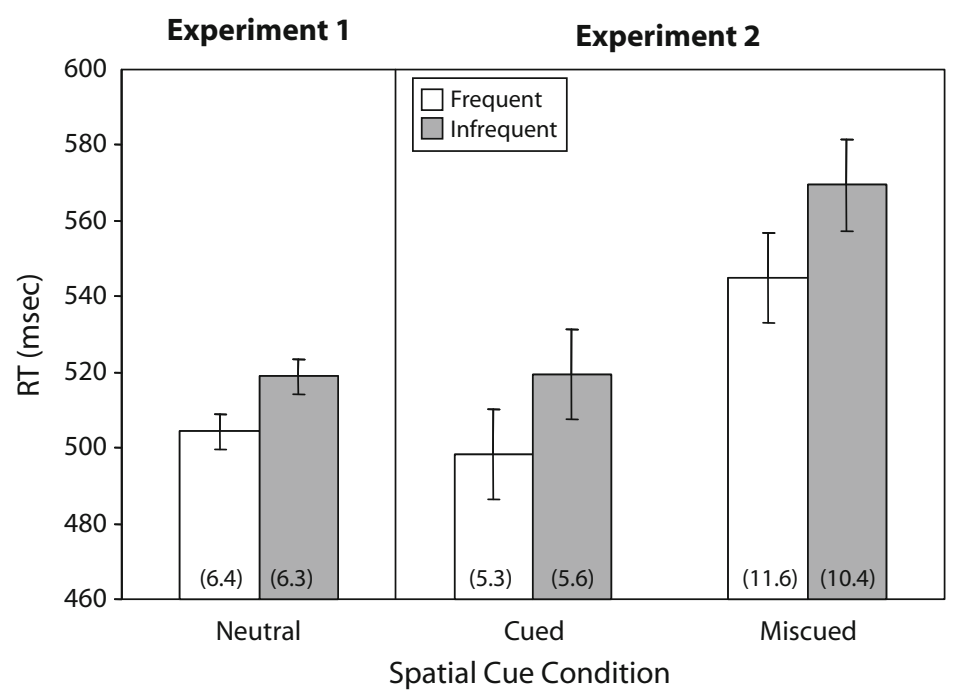

Figure 1. Response time (RT, in milliseconds) and percent error (in parentheses) as a function of cue-target event frequency and spatial cue condition in Experiments 1 (left panel) and 2 (right panel). Confidence intervals are based on the $M S_{\mathrm{e}}$ for the main effect of frequency (Masson \& Loftus, 2003). 
half of the participants, the spatial cue was presented at the top for $75 \%$ of the trials and at the bottom for $25 \%$ of the trials. The other half of the participants received the reverse order of presentation. On spatially cued trials, the spatial cue and the target appeared in the same location. On spatially miscued trials, the spatial cue and the target appeared in opposite locations. Spatially cued and miscued trials occurred equally often.

Stimuli. A spatial cue appeared $3.6 \mathrm{~cm}$ above or below fixation and consisted of a silver rectangle that subtended $1.5 \mathrm{~cm}$ horizontally and $1.0 \mathrm{~cm}$ vertically. All other characteristics of the stimuli, apparatus, and procedure are the same as in Experiment 1.

\section{Results}

The trimming procedure resulted in $2.9 \%$ of the correct RT data being discarded. The condition means from Experiment 2 are presented in the right panel of Figure 2.

A 2 (frequency) $\times 2$ (spatial cue condition) withinsubjects ANOVA was conducted on both RTs and percent error. Both the main effect of frequency $[F(1,39)=14.69$, $\left.M S_{\mathrm{e}}=1,419.36, p<.05\right]$ and the main effect of spatial cue condition $\left[F(1,39)=37.58, M S_{\mathrm{e}}=2,490.76, p<.05\right]$ were significant. Responses to frequent cue-target events (522 $\mathrm{msec})$ were faster than responses to infrequent cuetarget events (544 msec). Thirty participants demonstrated this cue-target event frequency effect. Responses to spatially cued trials $(509 \mathrm{msec})$ were faster than responses to spatially miscued trials $(557 \mathrm{msec})$. There was no interaction between frequency and spatial cue condition $(F<1)$. A planned $t$ test on spatially cued trials confirms the prediction made by the compound cue account: Responses to frequent cue-target events $(498 \mathrm{msec})$ were faster than responses to infrequent cue-target events (519 $\mathrm{msec}$ ) $[t(39)=2.76, p<.05]$. In percent error, there was a main effect of spatial cue condition $\left[F(1,39)=35.94, M S_{\mathrm{e}}=\right.$ $34.63, p<.05]$ such that percent error was lower on spatially cued trials $(5.4 \%)$ than on spatially miscued trials $(11.0 \%)$. There was no main effect of cue-target event frequency and no interaction between spatial cue condition and event frequency $\left(F_{\mathrm{S}}<1\right)$.

\section{Discussion}

Experiment 2 sought to discriminate between two alternative accounts of the neutral-cue frequency effect: a compound-cue memory-based account and an attention capture account. The results were clear. On spatially cued trials, responses to frequent cue-target events were faster than responses to infrequent cue-target events. This result is predicted by the memory-based account, whereas the opposite pattern is predicted by the attention capture account. The additivity between spatial cuing with an exogenous cue and cue frequency reported here is also consistent with Klein (1994), who showed that peripheral cuing is additive with target frequency. Thus, Experiment 2 provides further evidence that the frequency of a given cuetarget event can influence performance.

Immediate cue repetition. Increasing the frequency of a given event increases the likelihood that it will be repeated (Kornblum, 1969). Thus, with frequent cue-target events there are more spatial cue repetitions than with infrequent cue-target events. Repeated events are typically responded to faster than nonrepeated events. Thus, the advantage for frequent cue-target events reported here could be explicable in terms of a cue repetition effect. If spatial cue repetitions are responded to faster than spatial cue nonrepetitions, then the neutral-cue frequency effect may simply reflect this uneven distribution. To test this alternative hypothesis, analyses from Experiments 1 and 2 were repeated on a subset of trials in which the spatial cue did not repeat on consecutive trials (whether the target repeated or not was ignored). If a cue-target event frequency effect remains, it is not explicable in terms of the uneven distribution of spatial cue repetitions. Critically, frequent cue-target events were responded to faster than infrequent cue-target events for this subset of trials in both Experiment 1 (508 vs. $518 \mathrm{msec})[t(47)=2.42, p<.05]$ and Experiment 2 (525 vs. $547 \mathrm{msec})[t(39)=3.01, p<.05]$. Thus, the uneven distribution of spatial cue repetitions across frequent and infrequent cue-target events cannot explain the results of Experiments 1 and 2.

\section{GENERAL DISCUSSION}

We proposed a compound-cue frequency account of the proportion cued effect in the context of the covert orienting paradigm. Two experiments verified a central prediction of this account-specifically, that the cuetarget event acts as a compound cue and the frequency of occurrence of that compound cue affects performance. This account provides a parsimonious explanation of the proportion cued effect in the context of the covert orienting paradigm. Rather than attribute the proportion cued effect to an unspecified control mechanism, it is grounded in the well-accepted observation that frequent events are responded to faster than infrequent events.

The compound cue frequency account also offers an explanation for at least one aspect of the proportion cued effect that is not easily explicable in terms of control. Specifically, the proportion cued effect occurs independently of whether the participant is aware that the spatial cue is informative (Bartolomeo, Decaix, \& Sieroff, 2007). If the proportion cued effect reflects the strategic control of attention when the cue is spatially predictive, it would seem to require that participants be aware that the cue is spatially predictive (see also Bodner \& Masson, 2003). Recent research has demonstrated this not to be the case in the covert orienting paradigm (Bartolomeo et al., 2007). Importantly, the compound cue frequency account does not posit an explicit strategy on behalf of the participant.

It is important to note that the compound-cue account and the control account of the proportion cued effect are not mutually exclusive. The results of the present experiments only demonstrate that the compound-cue account is viable; they do not falsify the control account. Indeed, it is possible that only part of the proportion cued effect reflects the action of a compound cue retrieval process. ${ }^{2}$ In addition, it is possible that the proportion cued effect in different contexts reflects each mechanism to differing degrees. For example, in the present experiments a short cue-target interval $(150 \mathrm{msec})$ was used. It may be more likely that the spatial cue and the target form a compound cue in this context than in a context where a long cue-target interval (e.g., $800 \mathrm{msec}$ ) is used. 
Indeed, the fact that proportion cued effects occur with short cue-target intervals (see, e.g., Enns \& Brodeur, 1989) may in and of itself be considered inconsistent with the control account, given that "control" is typically thought to be slowacting (Posner \& Snyder, 1975).

If the proportion cued effect reflects, even in part, a compound cue frequency effect, then results from research using the proportion cued effect as a proxy for the control of visual attention would need to be reexamined (Danckert et al., 1998; Enns \& Brodeur, 1989; Maruff et al., 1998). Specifically, does the absence of a proportion cued effect reflect a "high-level" deficit in strategic control, as is typically argued, or some "low-level" deficit in the formation of a compound cue? These and other interesting questions emerge from viewing the proportion cued effect from the vantage point afforded by the compound cue frequency account proposed here.

\section{Conclusion}

The present investigation proposed a compound-cue frequency account of the proportion cued effect. The presentation of a demonstrably viable alternative to the control account of the proportion cued effect thus provides a heretofore absent challenge to the received view of this well-known effect. Future work contrasting these two accounts promises to advance our understanding of the mechanisms underlying visual attention.

\section{AUTHOR NOTE}

This work was supported by a Natural Sciences and Engineering Research Council of Canada Canadian Graduate Scholarship to E.F.R. and operating grants to J.A.S. and D.B. We thank Raymond Klein and Jim Neely for their helpful comments. Correspondence concerning this article should be addressed to E. F. Risko, Psychology Department, University of Waterloo, Waterloo, ON, N2L 3G1 Canada (e-mail: efrisko@ watarts.uwaterloo.ca).

\section{REFERENCES}

Bartolomeo, P., Decaix, C., \& Sieroff, E. (2007). The phenomenology of endogenous orienting. Consciousness \& Cognition, 16, 144-161.

Bertelson, P., \& Tisseyre, F. (1965). Choice reaction time as a function of stimulus versus response relative frequency of occurrence. Nature, 212, 1069-1070.

Bodner, G. E., \& MAsson, M. (2003). Beyond spreading activation: An influence of relatedness proportion on masked semantic priming. Psychonomic Bulletin \& Review, 10, 645-652.

COHEN, J. (1988). Statistical power analyses for the behavioral sciences. Hillsdale, NJ: Erlbaum.

Danckert, J., Maruff, P., Crowe, S., \& Currie, J. (1998). Inhibitory processes in covert orienting in patients with Alzheimer's disease. Neuropsychology, 12, 225-241.

Dosher, B. A., \& Rosedale, G. S. (1997). Configural processing in memory retrieval: Multiple cues and ensemble representations. Cognitive Psychology, 33, 209-265.

EnNs, J. T., \& BRodeur, D. A. (1989). A developmental study of covert orienting to peripheral visual cues. Journal of Experimental Child Psychology, 48, 171-189.
ERIKSEN, C. W., \& YeH, Y. Y. (1985). Allocation of attention in the visual field. Journal of Experimental Psychology: Human Perception \& Performance, 11, 583-587.

HyMAN, R. (1953). Stimulus information as a determinant of reaction time. Journal of Experimental Psychology, 45, 188-196.

Johnson, D. N., \& YANTIS, S. (1995). Allocating visual attention: Tests of a two-process model. Journal of Experimental Psychology: Human Perception \& Performance, 21, 1376-1390.

Kingstone, A. (1992). Combining expectancies. Quarterly Journal of Experimental Psychology, 44A, 69-104.

Kingstone, A., \& Klein, R. (1991). Combining shape and position expectancies: Hierarchical processing of selective inhibition. Journal of Experimental Psychology: Human Perception \& Performance, 17, 512-519.

KLeIN, R. M. (1994). Perceptual-motor expectancies interact with covert visual orienting under conditions of endogenous but not exogenous control. Canadian Journal of Experimental Psychology, 48, 167-181.

Kornblum, S. (1969). Sequential determinants of information processing in serial and discrete choice reaction time. Psychological Review, 76, 113-131.

LaBerge, D., Legrand, R., \& Hobbie, R. K. (1969). Functional identification of perceptual and response biases in choice reaction time. Journal of Experimental Psychology, 79, 295-299.

Logan, G. (1988). Towards an instance theory of automatization. Psychological Review, 95, 492-527.

MADDEN, D. J. (1992). Selective attention and visual search: Revision of an allocation model and application to age differences. Journal of Experimental Psychology: Human Perception \& Performance, 18, 821-836.

Maruff, P., Danckert, J., Pantelis, C., \& Currie, J. (1998). Saccadic and attentional abnormalities in patients with schizophrenia. Psychological Medicine, 28, 1091-1100.

Masson, M. E. J., \& LofTus, G. R. (2003). Using confidence intervals for graphically based data interpretation. Canadian Journal of Experimental Psychology, 57, 203-220.

Posner, M. I., Nissen, M. J., \& Ogden, W. C. (1978). Attended and unattended processing modes: The role of set for spatial location. In H. L. Pick \& I. J. Saltzman (Eds.), Modes of perceiving and processing information (pp. 137-157). Hillsdale, NJ: Erlbaum.

Posner, M. I., \& Petersen, S. E. (1990). The attention system of the human brain. Annual Review of Neurosciences, 13, 25-42.

Posner, M. I., \& SNYDER, C. R. R. (1975). Attention and cognitive control. In R.L. Solso (Ed.), Information processing and cognition: The Loyola symposium (pp. 55-85). Hillsdale, NJ: Erlbaum.

Psychology Software Tools (2002). E-Prime (Version 1.1) [Computer software]. Pittsburgh: Psychology Software Tools.

Ratcliff, R., \& McKoon, G. (1988). A retrieval theory of priming in memory. Psychological Review, 95, 385-408.

VAn SElst, M., \& Joliceurr, P. (1994). A solution to the effect of sample size on outlier elimination. Quarterly Journal of Experimental Psychology, 47A, 631-650.

\section{NOTES}

1. We replicated Experiment 1 using a detection task rather than an identification task and found that responses to frequent cue-target events $(313 \mathrm{msec})$ were, again, faster than responses to infrequent cue-target events $(320 \mathrm{msec})\left[F(1,19)=8.26, M S_{\mathrm{e}}=67.33, p<.05\right]$.

2. In terms of effect size (Cohen, 1988), the frequency effects in Experiments $1(.62)$ and $2(.60)$ are somewhat smaller than the effect size we obtained in an unpublished study from our lab using the same stimulus parameters and comparing the magnitude of the cuing effect across a $50 \%$ and a $75 \%$ cued condition (.82).

(Manuscript received March 23, 2007; revision accepted for publication June 13, 2007.) 